\title{
Multiple homoclinic orbits for second order discrete Hamiltonian systems without symmetric condition
}

\section{Zhimin $\mathrm{He}^{1 *}$ and Huiwen Chen ${ }^{2}$}

"Correspondence:

hezhimin@csu.edu.cn

'School of Mathematics and

Statistics, Central South University,

Changsha, Hunan 410083, People's

Republic of China

Full list of author information is

available at the end of the article

\begin{abstract}
In this paper, we investigate the second order self-adjoint discrete Hamiltonian system $\Delta[p(n) \Delta u(n-1)]-L(n) u(n)+\lambda a(n) \nabla G(u(n))+\mu b(n) \nabla F(u(n))=0$, where $p, L: \mathbb{Z} \rightarrow \mathbb{R}^{N \times N}$ are both positive definite for all $n \in \mathbb{Z}$, and no symmetric condition on $G$ and $F$ is needed. We establish two new criteria to guarantee that the above system has at least two nontrivial homoclinic solutions or infinitely many homoclinic solutions via critical point theory.
\end{abstract}

MSC: 37J45; 39A 12; 58E05; 70H05

Keywords: homoclinic solutions; discrete Hamiltonian systems; critical point theory; variational methods

\section{Introduction}

In this paper, we consider the following second order self-adjoint discrete Hamiltonian system:

$$
\Delta[p(n) \Delta u(n-1)]-L(n) u(n)+\lambda a(n) \nabla G(u(n))+\mu b(n) \nabla F(u(n))=0, \quad \forall n \in \mathbb{Z},
$$

where $u \in \mathbb{R}^{N}, \Delta u(n)=u(n+1)-u(n)$ is the forward difference, $p, L: \mathbb{Z} \rightarrow \mathbb{R}^{N \times N}$. As usual, we say that a solution $u(n)$ of system (1.1) is homoclinic (to 0$)$ if $u(n) \rightarrow 0$ as $|n| \rightarrow \infty$. In addition, if $u(n) \not \equiv 0$ then $u(n)$ is called a nontrivial homoclinic solution.

The discrete Hamiltonian system has found a great deal of interest last years because not only it is important in applications but it provides a good model for developing mathematical methods. In general, system (1.1) may be regarded as a discrete analog of the following second order Hamiltonian system:

$$
\frac{d}{d t}(p(t) \dot{u}(t))-L(t) u(t)+\lambda a(t) \nabla G(u(t))+\mu b(t) \nabla F(u(t))=0 .
$$

With the aid of variational methods, the existence and multiplicity of homoclinic orbits for system (1.2) or its special form has been extensively investigated in many recent papers; see [1-16]. System (1.1) is the best approximation of (1.2) when one lets the step size not be equal to 1 but the variable's step size go to 0 , so solutions of system (1.1) can give some

(c) $2015 \mathrm{He}$ and Chen. This article is distributed under the terms of the Creative Commons Attribution 4.0 International License (http://creativecommons.org/licenses/by/4.0/), which permits unrestricted use, distribution, and reproduction in any medium, provided you give appropriate credit to the original author(s) and the source, provide a link to the Creative Commons license, and indicate if changes were made. 
desirable numerical features for system (1.2). Moreover, system (1.1) does have applications as is shown in the monographs $[17,18]$.

In the past ten years, many authors have studied the existence and multiplicity of homoclinic solutions for system (1.1) or its special form (with $F=0$ ) via variational methods; see [19-31] and the references therein. In particular, see [20, 22, 24, 25, 28, 29, 31]. The authors have studied the existence of multiple homoclinic solutions for system (1.1) under the assumption that the nonlinear term is symmetric.

When no symmetric condition on the nonlinear term is assumed, as far as the authors are aware, there is no research about the existence of multiple homoclinic solutions for system (1.1). Motivated by the above facts, in this paper, our aim is to study the existence of multiple homoclinic solutions for system (1.1) under the condition that the nonlinear term possesses no symmetric condition.

We will use the following conditions:

$\left(\mathrm{F}_{1}\right) \quad p(n)$ is a real symmetric positive definite matrix for all $n \in \mathbb{Z}$.

$\left(\mathrm{F}_{2}\right) \quad L(n)$ is a real symmetric positive definite matrix for all $n \in \mathbb{Z}$ and

$$
l(n)=\inf _{|u|=1}(L(n) u, u) \rightarrow+\infty
$$

as $|n| \rightarrow \infty$.

(F $\left.\mathrm{F}_{3}\right) \quad G \in C^{1}\left(\mathbb{R}^{N}, \mathbb{R}\right)$ and $G(0)=0$.

$\left(\mathrm{F}_{4}\right) \quad a \in l^{\frac{2}{1-\gamma}}(\mathbb{Z}, \mathbb{R})$ is a non-negative, non-zero function (for some $\gamma \in(0,1)$ ).

(F) $\lim _{u \rightarrow 0} \frac{|\nabla G(u)|}{|u|}=0$.

(F) $\lim _{|u| \rightarrow \infty} \frac{|\nabla G(u)|}{|u|}=0$.

$\left(\mathrm{F}_{7}\right)$ There exists $\zeta=\left(\zeta_{1}, \zeta_{2}, \ldots, \zeta_{N}\right) \in \mathbb{R}^{N}$ such that $G(\zeta)>0$.

Denote by $\Lambda$ the set of functions $F \in C^{1}\left(\mathbb{R}^{N}, \mathbb{R}\right)$, such that $F(0)=0$ and satisfying the property:

( $\left.\mathrm{F}_{8}\right)$ There exist $d>0$ and $\alpha>1$ such that

$$
|\nabla F(u)| \leq d\left(|u|+|u|^{\alpha}\right), \quad \forall u \in \mathbb{R}^{N} .
$$

( $\left.\mathrm{F}_{9}\right) \quad a \in l^{1}(\mathbb{Z}, \mathbb{R})$ is a non-negative, non-zero function.

$\left(\mathrm{F}_{10}\right) \frac{\|a\|_{1}}{\sqrt{\left(\varrho_{2}+4 \varrho_{1}\right) \varrho_{2}}} \liminf _{\eta \rightarrow \infty} \frac{\max _{|u| \leq \eta} G(u)}{\eta^{2}}<\frac{a\left(n_{0}\right)}{2 l_{1}+l_{2}} \lim \sup _{|u| \rightarrow \infty} \frac{G(u)}{|u|^{2}}$, where $n_{0} \in \mathbb{Z}$ with $a\left(n_{0}\right)=$ $\max \{a(n): n \in \mathbb{Z}\}, \varrho_{1}=\inf \left\{(p(n) x, x): n \in \mathbb{Z}, x \in \mathbb{R}^{N},|x|=1\right\}, \varrho_{2}=\inf \{l(n): n \in \mathbb{Z}\}, l_{1}=$ $\sup \left\{(p(n) x, x): n=n_{0}, n_{0}+1, x \in \mathbb{R}^{N},|x|=1\right\}$ and $l_{2}=\sup \left\{\left(L\left(n_{0}\right) x, x\right): x \in \mathbb{R}^{N},|x|=1\right\}$.

$\left(\mathrm{F}_{11}\right)$ There exist $M>0$ and $v>2$ such that

$$
|\nabla G(u)| \leq M\left(|u|+|u|^{\nu-1}\right), \quad \forall u \in \mathbb{R}^{N} .
$$

Now, we state our main results.

Theorem 1.1 Assume that $\left(\mathrm{F}_{1}\right)-\left(\mathrm{F}_{7}\right)$ hold. Then there exists $\lambda_{1}>0$ such that for each $\lambda>\lambda_{1}$, and for every function $b \in l^{\infty}(\mathbb{Z}, \mathbb{R})$ and every function $F \in \Lambda$, there is $\sigma>0$ with property that for each $\mu \in[0, \sigma]$, system (1.1) has at least two nontrivial homoclinic solutions.

Example 1.1 $a(n)=\left(\frac{1}{1+n^{2}}\right)^{1 / 4}, G(u)=\min \left\{|u|^{\sigma_{1}},|u|^{\sigma_{2}}\right\}$ with $1<\sigma_{1}<2<\sigma_{2}$. 
Example 1.2 $a(n)=\left(\frac{1}{1+n^{2}}\right)^{1 / 4}, G(u)=((1,1, \ldots, 1), u) \ln \left(1+|u|^{2}\right)$. It is clear that $G(u)$ is not even.

Remark 1.1 In Example 1.1 and Example 1.2, one cannot obtain the existence of homoclinic solutions in $[20,22,24,25,28,29,31]$. But in this paper, we obtain the existence of two homoclinic solutions.

When $F=0$, a new result is obtained.

Theorem 1.2 Assume that $\left(\mathrm{F}_{1}\right)-\left(\mathrm{F}_{3}\right)$ and $\left(\mathrm{F}_{9}\right)-\left(\mathrm{F}_{11}\right)$ hold. Then, for every

$$
\lambda \in \Omega:=\left(\frac{2 l_{1}+l_{2}}{2 a\left(n_{0}\right) \lim \sup _{|u| \rightarrow \infty} \frac{G(u)}{|u|^{2}}}, \frac{\sqrt{\left(\varrho_{2}+4 \varrho_{1}\right) \varrho_{2}}}{2\|a\|_{1} \liminf _{\eta \rightarrow \infty} \frac{\max |u| \leq \eta}{\eta^{2}} G(u)}\right),
$$

system (1.1) (with $F=0)$ possesses an unbounded sequence of homoclinic solutions.

Remark 1.2 In Theorem 1.2, we can substitute $\eta \rightarrow \infty$ and $|u| \rightarrow \infty$ with $\eta \rightarrow 0^{+}$and $|u| \rightarrow 0^{+}$by applying part (c) of Theorem 2.2 instead of part (b) of Theorem 2.2 in the proof, and obtaining a sequence of pairwise distinct homoclinic solutions.

The remainder of this paper is organized as follows. In Section 2, some preliminary results are presented. In Section 3, we give the proof of Theorems 1.1 and 1.2.

\section{Preliminaries}

In this section, the following Ricceri-type three critical points theorem will be needed in our argument. If $X$ is a real Banach space, denote by $\Gamma_{X}$ the class of all functionals $\psi: X \rightarrow \mathbb{R}$ possessing the following property: if $\left\{u_{n}\right\}$ is a sequence in $X$ converging weakly to $u \in X$ and $\liminf _{n \rightarrow \infty} \psi\left(u_{n}\right) \leq \psi(u)$, then $\left\{u_{n}\right\}$ has a subsequence converging strongly to $u$.

For example, if $X$ is uniformly convex and $g:[0,+\infty) \rightarrow \mathbb{R}$ is a continuous, strictly increasing function, then, by a classical result, the functional $u \rightarrow g(\|u\|)$ belongs to the class $\Gamma_{X}$.

Theorem 2.1 ([32], Theorem 2) Let $X$ be a separable and reflexive real Banach space; let $\Phi: X \rightarrow \mathbb{R}$ be a coercive, sequentially weakly lower semicontinuous $C^{1}$ functional, belonging to $\Gamma_{X}$, bounded on each bounded subset of $X$ and whose derivative admits a continuous inverse on $X^{*} ; J: X \rightarrow \mathbb{R}$ a $C^{1}$ functional with compact derivative. Assume that $\Phi$ has a strict local minimum $v_{0}$ with $\Phi\left(v_{0}\right)=J\left(v_{0}\right)=0$. Finally, setting

$$
\begin{aligned}
& \alpha_{1}=\max \left\{0, \limsup _{\|v\| \rightarrow+\infty} \frac{J(v)}{\Phi(v)}, \limsup _{v \rightarrow v_{0}} \frac{J(v)}{\Phi(v)}\right\}, \\
& \alpha_{2}=\sup _{v \in \Phi^{-1}((0,+\infty))} \frac{J(v)}{\Phi(v)},
\end{aligned}
$$

assume that $\alpha_{1}<\alpha_{2}$.

Then, for each compact interval $\left[b_{1}, b_{2}\right] \subset\left(\frac{1}{\alpha_{2}}, \frac{1}{\alpha_{1}}\right)$ (with the conventions $\frac{1}{0}=+\infty, \frac{1}{+\infty}=0$ ), there exists $N_{1}>0$ with the following property: for every $\lambda \in\left[b_{1}, b_{2}\right]$ and every $C^{1}$ functional 
$\Psi: X \rightarrow \mathbb{R}$ with compact derivative, there exists $\sigma>0$ such that, for each $\mu \in[0, \sigma]$, the equation

$$
\Phi^{\prime}(v)=\lambda J^{\prime}(v)+\mu \Psi^{\prime}(v)
$$

has at least three solutions in $X$ whose norms are less than $N_{1}$.

In the next section we shall prove our results applying the classical Ricceri variational principle ([33], Theorem 2.5).

Let $X$ be a non-empty set and let $\Phi, J: X \rightarrow \mathbb{R}$ be two functionals.

For all $r>\inf _{X} \Phi$, we put

$$
\varphi_{1}(r)=\inf _{u \in \Phi^{-1}((-\infty, r))} \frac{\sup _{v \in \Phi^{-1}((-\infty, r))} J(v)-J(u)}{r-\Phi(u)}
$$

and

$$
\gamma_{1}:=\liminf _{r \rightarrow+\infty} \varphi_{1}(r), \gamma_{2}:=\liminf _{\left.r \rightarrow \inf _{X} \Phi\right)^{+}} \varphi_{1}(r)
$$

Theorem 2.2 ([33], Theorem 2.5) Let $X$ be a reflexive real Banach space, and let $\Phi, J$ : $X \rightarrow \mathbb{R}$ be two Gâteaux differentiable functionals such that $\Phi$ is sequentially weakly lower semicontinuous, (strongly) continuous, and coercive and $J$ is sequentially weakly continuous. One has:

(a) For every $r>\inf _{X} \Phi$ and every $\lambda \in\left(0, \frac{1}{\varphi_{1}(r)}\right)$, the restriction of the functional $\Phi-\lambda J$ to $\Phi^{-1}((-\infty, r))$ admits a global minimum, which is a critical point (local minimum) of $\Phi-\lambda J$ in $X$.

(b) If $\gamma_{1}<+\infty$ then, for each $\lambda \in\left(0, \frac{1}{\gamma_{1}}\right)$, the following alternative holds: either

$\left(b_{1}\right) \Phi-\lambda \Psi$ possesses a global minimum, or

$\left(\mathrm{b}_{2}\right)$ there is a sequence $\left\{u_{n}\right\}$ of critical points (local minima) of $\Phi-\lambda J$ such that $\lim _{n \rightarrow+\infty} \Phi\left(u_{n}\right)=+\infty$.

(c) If $\gamma_{2}<+\infty$ then, for each $\lambda \in\left(0, \frac{1}{\gamma_{2}}\right)$, the following alternative holds: either

$\left(\mathrm{c}_{1}\right)$ there is a global minimum of $\Phi$ which is a local minimum of $\Phi-\lambda J$, or

$\left(\mathrm{c}_{2}\right)$ there is a sequence of pairwise distinct critical points (local minima) of $\Phi-\lambda J$ which weakly converges to a global minimum of $\Phi$.

In what follows, we always assume that $p(n)$ and $L(n)$ are real symmetric positive definite matrices for all $n \in \mathbb{Z}$. Let

$$
\begin{aligned}
& H=\left\{\{u(n)\}_{n \in \mathbb{Z}}: u(n) \in \mathbb{R}^{N}, n \in \mathbb{Z}\right\}, \\
& X=\left\{u \in H: \sum_{n \in \mathbb{Z}}[(p(n+1) \Delta u(n), \Delta u(n))+(L(n) u(n), u(n))]<+\infty\right\}
\end{aligned}
$$

and for $u, v \in X$, let

$$
\langle u, v\rangle=\sum_{n \in \mathbb{Z}}[(p(n+1) \Delta u(n), \Delta v(n))+(L(n) u(n), v(n))],
$$


and the corresponding norm is

$$
\|u\|=\left\{\sum_{n \in \mathbb{Z}}[(p(n+1) \Delta u(n), \Delta u(n))+(L(n) u(n), u(n))]\right\}^{\frac{1}{2}}, \quad \forall u \in X .
$$

Then $X$ is a Hilbert space with the above inner product. As usual, for $1 \leq q<+\infty, j=$ 1 or $N$, let

$$
l^{q}\left(\mathbb{Z}, \mathbb{R}^{j}\right)=\left\{\{u(n)\}_{n \in \mathbb{Z}}: u(n) \in \mathbb{R}^{j}, n \in \mathbb{Z}, \sum_{n \in \mathbb{Z}}|u(n)|^{q}<+\infty\right\}
$$

and

$$
l^{\infty}\left(\mathbb{Z}, \mathbb{R}^{j}\right)=\left\{\{u(n)\}_{n \in \mathbb{Z}}: u(n) \in \mathbb{R}^{j}, n \in \mathbb{Z}, \sup _{n \in \mathbb{Z}}|u(n)|<+\infty\right\}
$$

and their norms are defined by

$$
\begin{aligned}
\|u\|_{q} & =\left(\sum_{n \in \mathbb{Z}}|u(n)|^{q}\right)^{\frac{1}{q}}, \quad \forall u \in l^{q}\left(\mathbb{Z}, \mathbb{R}^{j}\right) ; \\
\|u\|_{\infty} & =\sup _{n \in \mathbb{Z}}|u(n)|, \quad \forall u \in l^{\infty}\left(\mathbb{Z}, \mathbb{R}^{j}\right),
\end{aligned}
$$

respectively.

Lemma 2.1 (see [28]) For $u \in X$,

$$
\|u\|_{\infty} \leq \frac{1}{\sqrt[4]{\left(\varrho_{2}+4 \varrho_{1}\right) \varrho_{2}}}\|u\|
$$

where $\varrho_{1}=\inf \left\{(p(n) x, x): n \in \mathbb{Z}, x \in \mathbb{R}^{N},|x|=1\right\}$ and $\varrho_{2}=\inf \{l(n): n \in \mathbb{Z}\}$ and, for $u \in$ $l^{2}\left(\mathbb{Z}, \mathbb{R}^{N}\right)$,

$$
\|u\|_{\infty} \leq \frac{1}{\sqrt[4]{5}}\left\{\sum_{n \in \mathbb{Z}}\left[|\Delta u(n)|^{2}+|u(n)|^{2}\right]\right\}^{\frac{1}{2}} .
$$

Lemma 2.2 (see [29]) Assume that $L$ satisfies $\left(\mathrm{F}_{2}\right)$. Then $X$ is compactly embedded in $l^{q}\left(\mathbb{Z}, \mathbb{R}^{N}\right)$ for any $2 \leq q<\infty$, and

$$
\begin{aligned}
& \|u\|_{q}^{q} \leq \varrho_{2}^{-1}\left[\left(\varrho_{2}+4 \varrho_{1}\right) \varrho_{2}\right]^{\frac{2-q}{4}}\|u\|^{q}, \quad \forall u \in X, \\
& \sum_{|n|>N_{2}}|u(n)|^{q} \leq \frac{\left[\left(\varrho_{2}+4 \varrho_{1}\right) \varrho_{2}\right]^{\frac{2-q}{4}}}{\min _{|n| \geq N_{2}} l(n)}\|u\|^{q}, \quad \forall u \in X, N_{2} \geq 1 .
\end{aligned}
$$

For any $u \in X$, put

$$
\Phi(u)=\frac{1}{2}\|u\|^{2}, \quad J(u)=\sum_{n \in \mathbb{Z}} a(n) G(u(n)), \quad \Psi(u)=\sum_{n \in \mathbb{Z}} b(n) F(u(n)) .
$$


Lemma 2.3 Assume that $\left(\mathrm{F}_{1}\right)-\left(\mathrm{F}_{8}\right)$ hold. Then the functional $I: X \rightarrow \mathbb{R}$ defined by

$$
\begin{aligned}
I(u) & =\frac{1}{2}\|u\|^{2}-\lambda \sum_{n \in \mathbb{Z}} a(n) G(u(n))-\mu \sum_{n \in \mathbb{Z}} b(n) F(u(n)) \\
& =\Phi(u)-\lambda J(u)-\mu \Psi(u), \quad \forall u \in E,
\end{aligned}
$$

is well defined and of class $C^{1}(X, \mathbb{R})$ and

$$
\begin{aligned}
\left\langle I^{\prime}(u), v\right\rangle= & \sum_{n \in \mathbb{Z}}[(p(n+1) \Delta u(n), \Delta v(n))+(L(n) u(n), v(n))] \\
& -\sum_{n \in \mathbb{Z}}[\lambda(a(n) \nabla G(u(n)), v(n))+\mu(b(n) \nabla G(u(n)), v(n))], \quad \forall u, v \in X .
\end{aligned}
$$

Furthermore, $J^{\prime}, \Psi^{\prime}: X \rightarrow X^{*}$ are compact and the critical points of I in $X$ are solutions of system (1.1) with $u( \pm \infty)=0$.

Proof We first show that $J: X \rightarrow \mathbb{R}$. It follows from $\left(\mathrm{F}_{5}\right)$ and $\left(\mathrm{F}_{6}\right)$ that for any $\epsilon>0$, there exists $D_{\epsilon}$ such that

$$
|\nabla G(u)| \leq \epsilon|u|+D_{\epsilon}|u|^{2} .
$$

By $(2.8),\left(\mathrm{F}_{4}\right)$, and (2.3), we have

$$
\begin{aligned}
\left|\sum_{n \in \mathbb{Z}} a(n) G(u(n))\right| & \leq\|a\|_{\infty}\left(\epsilon \sum_{n \in \mathbb{Z}}|u(n)|^{2}+D_{\epsilon} \sum_{n \in \mathbb{Z}}|u(n)|^{3}\right) \\
& \leq D\left(\|u\|^{2}+\|u\|^{3}\right)
\end{aligned}
$$

for some $D>0$. Analogously, we see that $\Psi$ is well defined on $X$. Thus, $I$ is well defined on $X$.

Now, we show that $J$ is Gâteaux differentiable on $X$. By virtue of $(2.8)$ and $\left(\mathrm{F}_{4}\right)$, for any $\xi \in[0,1]$, it is easy to check that

$$
|(a(n) \nabla G(u+\xi v), v)| \leq C\left(|u|^{2}+|v|^{2}+|u|^{4}+|v|^{3}\right), \quad \forall n \in \mathbb{Z} \text { and } u, v \in \mathbb{R}^{N},
$$

where $C$ is a constant independent of $\xi$. Therefore, for any $u, v \in X$, by the mean value theorem and the Lebesgue dominated convergence theorem, we have

$$
\begin{aligned}
\lim _{h \rightarrow 0} \frac{J(u+h v)-J(u)}{h} & =\lim _{h \rightarrow 0} \sum_{n \in \mathbb{Z}}\left(a(n) \nabla G\left(u(n)+\theta_{n} h v(n)\right), v\right) \\
& =\sum_{n \in \mathbb{Z}}(a(n) \nabla G(u(n)), v(n)) \\
& =: W(u, v),
\end{aligned}
$$

where $\theta_{n} \in[0,1]$ depends on $u, v, h$. It is easy to see that $W(u, v)$ is linear. Next we show that $W(u, v)$ is bounded. In fact, for any $u \in X$, by $\left(\mathrm{F}_{3}\right),\left(\mathrm{F}_{4}\right),(2.3)$, and Hölder's inequality, 
we have

$$
\begin{aligned}
|W(u, v)| & =\left|\sum_{n \in \mathbb{Z}}(a(n) \nabla G(u(n)), v(n))\right| \\
& \leq \sum_{n \in \mathbb{Z}}|a(n) \nabla G(u(n))||v(n)| \\
& \leq\|a\|_{\infty} \sum_{n \in \mathbb{Z}}\left(\epsilon|u(n)|+D_{\epsilon}|u(n)|^{2}\right)|v(n)| \\
& \leq C_{1}\left(\|u\|+\|u\|^{2}\right)\|v\|
\end{aligned}
$$

for some $C_{1}>0$. Therefore, $D J(u)=W(u, \cdot)$ is the Gâteaux derivative of $J$ at $u$.

Next, we prove that $D J(u)$ is weakly continuous in $u$. To this end, we first claim that if $u_{k} \rightarrow u$ in $X$, then $f_{k} \rightarrow f$ in $l^{2}\left(\mathbb{Z}, \mathbb{R}^{N}\right)$, where $f_{k}=\left\{\nabla G\left(u_{k}(n)\right)\right\}_{n \in \mathbb{Z}}$ and $f=\{\nabla G(u(n))\}_{n \in \mathbb{Z}}$. Arguing indirectly, by Lemma 2.2 , we may assume that there exists a subsequence $\left\{u_{k_{i}}\right\}$ such that

$$
\begin{aligned}
& u_{k_{i}} \rightarrow u \quad \text { in both } l^{2}\left(\mathbb{Z}, \mathbb{R}^{N}\right) \text { and } l^{4}\left(\mathbb{Z}, \mathbb{R}^{N}\right), \\
& u_{k_{i}}(n) \rightarrow u(n), \quad \forall n \in \mathbb{Z} \text { as } i \rightarrow \infty
\end{aligned}
$$

and

$$
\sum_{n \in \mathbb{Z}}\left|\nabla G\left(u_{k_{i}}(n)\right)-\nabla G(u(n))\right|^{2} \geq \varepsilon, \quad \forall i \in \mathbb{Z}
$$

for some $\varepsilon>0$. By (2.12), passing to a subsequence if necessary, we can assume that

$$
\sum_{i=1}^{\infty}\left\|u_{k_{i}}-u\right\|_{2}^{2}<+\infty
$$

and

$$
\sum_{i=1}^{\infty}\left\|u_{k_{i}}-u\right\|_{4}^{4}<+\infty
$$

Let

$$
e_{1}(n)=\left(\sum_{i=1}^{\infty}\left|u_{k_{i}}(n)-u(n)\right|^{2}\right)^{1 / 2}
$$

and

$$
e_{2}(n)=\left(\sum_{i=1}^{\infty}\left|u_{k_{i}}(n)-u(n)\right|^{4}\right)^{1 / 4},
$$

then $\left\{e_{1}(n)\right\}_{n \in \mathbb{Z}} \in l^{2}(\mathbb{Z}, \mathbb{R})$ and $\left\{e_{2}(n)\right\}_{n \in \mathbb{Z}} \in l^{4}(\mathbb{Z}, \mathbb{R})$. It follows from (2.8) that

$$
\begin{aligned}
& \left|\nabla G\left(u_{k_{i}}(n)\right)-\nabla G(u(n))\right|^{2} \\
& \quad \leq\left(\epsilon\left|u_{k_{i}}(n)\right|+\epsilon|u(n)|+D_{\epsilon}\left|u_{k_{i}}(n)\right|^{2}+D_{\epsilon}|u(n)|^{2}\right)^{2}
\end{aligned}
$$




$$
\begin{aligned}
& \leq\left(\epsilon\left|u_{k_{i}}(n)-u(n)\right|+2 \epsilon|u(n)|+2 D_{\epsilon}\left|u_{k_{i}}(n)-u(n)\right|^{2}+3 D_{\epsilon}|u(n)|^{2}\right)^{2} \\
& \leq 4 \epsilon^{2}\left(\left|u_{k_{i}}(n)-u(n)\right|^{2}+4|u(n)|^{2}\right)+4 D_{\epsilon}^{2}\left(4\left|u_{k_{i}}(n)-u(n)\right|^{4}+9|u(n)|^{4}\right) \\
& \leq 4 \epsilon^{2}\left(\left|e_{1}(n)\right|^{2}+4|u(n)|^{2}\right)+4 D_{\epsilon}^{2}\left(4\left|e_{2}(n)\right|^{4}+9|u(n)|^{4}\right)
\end{aligned}
$$

for all $i, n \in \mathbb{Z}$. Combining (2.12) and (2.14), by Lebesgue dominated convergence, we have

$$
\lim _{i \rightarrow \infty} \sum_{n \in \mathbb{Z}}\left|\nabla G\left(u_{k_{i}}(n)\right)-\nabla G(u(n))\right|^{2}=0,
$$

which contradicts (2.13). Hence the claim above is true. We assume that $u_{k} \rightarrow u$ in $X$, then $f_{k} \rightarrow f$ in $l^{2}\left(\mathbb{Z}, \mathbb{R}^{N}\right)$, where $f_{k}=\left\{\nabla G\left(u_{k}(n)\right)\right\}_{n \in \mathbb{Z}}$ and $f=\{\nabla G(u(n))\}_{n \in \mathbb{Z}}$. By $(2.3),\left(\mathrm{F}_{4}\right)$, and Hölder's inequality, we obtain

$$
\begin{aligned}
\left\|D J\left(u_{k}\right)-D J(u)\right\|_{X^{*}} & =\sup _{\|v\|=1}\left|\left\langle D J\left(u_{k}\right)-D J(u), v\right\rangle\right| \\
& =\sup _{\|\nu\|=1}\left|\sum_{n \in \mathbb{Z}}\left(a(n)\left(\nabla G\left(u_{k}(n)\right)-\nabla G(u(n))\right), v(n)\right)\right| \\
& \leq\|a\|_{\infty}\left(\sum_{n \in \mathbb{Z}}\left|\nabla G\left(u_{k}(n)\right)-\nabla G(u(n))\right|^{2}\right)^{\frac{1}{2}}\|v\|_{2} \\
& \leq \frac{1}{\sqrt{\varrho_{2}}}\|a\|_{\infty}\left(\sum_{n \in \mathbb{Z}}\left|\nabla G\left(u_{k}(n)\right)-\nabla G(u(n))\right|^{2}\right)^{\frac{1}{2}} \rightarrow 0
\end{aligned}
$$

as $k \rightarrow \infty$. This implies that $D J(u)$ is weakly continuous in $u$. Hence, $J^{\prime}(u)=D J(u)$, i.e., $J \in C^{1}(X, \mathbb{R})$. Furthermore, $J^{\prime}$ is compact by the weakly continuity of $J^{\prime}$ since $X$ is a Hilbert space. Similarly, we can prove that $\Psi \in C^{1}(X, \mathbb{R})$ and $\Psi^{\prime}$ is compact. Due to the form of $I$ in (2.6), (2.7) is verified and hence $I \in C^{1}(X, \mathbb{R})$.

Finally, we show that the critical points of $I$ in $X$ are solutions of system (1.1) with $u( \pm \infty)=0$. Observe that, for any $u, v \in X$,

$$
\begin{aligned}
\sum_{n \in \mathbb{Z}} & (p(n+1) \Delta u(n), \Delta v(n))+(L(n) u(n), v(n)) \\
& \quad-\lambda(a(n) \nabla G(u(n)), v(n))-\mu(b(n) \nabla F(u(n)), v(n))] \\
= & \sum_{n \in \mathbb{Z}}[(p(n+1) \Delta u(n), v(n+1))-(p(n+1) \Delta u(n), v(n))] \\
& \quad+\sum_{n \in \mathbb{Z}}[(L(n) u(n), v(n))-\lambda(a(n) \nabla G(u(n)), v(n))-\mu(b(n) \nabla F(u(n)), v(n))] \\
= & \sum_{n \in \mathbb{Z}}[(p(n) \Delta u(n-1), v(n))-(p(n+1) \Delta u(n), v(n))] \\
& \quad+\sum_{n \in \mathbb{Z}}[(L(n) u(n), v(n))-\lambda(a(n) \nabla G(u(n)), v(n))-\mu(b(n) \nabla F(u(n)), v(n))] \\
= & \sum_{n \in \mathbb{Z}}(-\Delta(p(n) \Delta u(n-1))+L(n) u(n)-\lambda a(n) \nabla G(u(n))-\mu b(n) \nabla F(u(n)), v(n)) .
\end{aligned}
$$

It follows from (2.7) and the above equations that $\left\langle I^{\prime}(u), v\right\rangle=0$ for all $v \in X$ if and only if

$$
\Delta[p(n) \Delta u(n-1)]-L(n) u(n)+\lambda a(n) \nabla G(u(n))+\mu b(n) \nabla F(u(n))=0, \quad \forall n \in \mathbb{Z} .
$$


So, the critical points of $I$ in $X$ are the solutions of system (1.1). On the other hand, it follows from (2.3) that $u( \pm \infty)=0$. This completes the proof.

Lemma 2.4 $\Phi$ is coercive, sequentially weakly lower semicontinuous, bounded on each bounded subset of $X$ and its derivative admits a continuous inverse on $X^{*}$.

Proof It is easy to verify that $\Phi$ is coercive. Let $u_{k} \rightarrow u$ in $X$, we see that $\liminf _{n \rightarrow \infty}\left\|u_{k}\right\| \geq$ $\|u\|$. Then we have

$$
\liminf _{n \rightarrow \infty} \Phi\left(u_{k}\right)=\liminf _{n \rightarrow \infty} \frac{1}{2}\left\|u_{k}\right\|^{2} \geq \frac{1}{2}\|u\|^{2}=\Phi(u) .
$$

So $\Phi$ is sequentially weakly lower semicontinuous. Moreover, it is easy to see that $\Phi$ is bounded on each bounded subset of $X$.

Next we will show that $\Phi^{\prime}$ admits a continuous inverse on $X^{*}$. For each $u \in X \backslash\{0\}$, by (2.7), we have

$$
\left\langle\Phi^{\prime}(u), u\right\rangle=\|u\|^{2} .
$$

So $\lim _{\|u\| \rightarrow+\infty}\left\langle\Phi^{\prime}(u), u\right\rangle /\|u\|=+\infty$, that is, $\Phi^{\prime}$ is coercive. For any $u, v \in X$, in view of (2.7), we have

$$
\left\langle\Phi^{\prime}(u)-\Phi^{\prime}(v), u-v\right\rangle=\|u-v\|^{2} .
$$

So $\Phi^{\prime}$ is uniformly monotone. By ([34], Theorem $26 . \mathrm{A}(\mathrm{d})$ ), we see that $\Phi^{\prime}$ admits a continuous inverse on $X^{*}$.

\section{Proof of Theorems 1.1 and 1.2}

We will prove Theorem 1.1 by using Theorem 2.1. First, we give the following four useful lemmas.

Lemma 3.1 $\lim \sup _{u \rightarrow 0} \frac{J(u)}{\Phi(u)} \leq 0$.

Proof It follows from $\left(\mathrm{F}_{3}\right),\left(\mathrm{F}_{5}\right)$, and $\left(\mathrm{F}_{6}\right)$ that, for any $\epsilon>0$, there exists $T_{\epsilon}$ such that

$$
|G(u)| \leq \frac{\epsilon}{4\left(1+\|a\|_{\infty}\right)}|u|^{2}+T_{\epsilon}|u|^{3} .
$$

By (3.1) and (2.3), one has

$$
\begin{aligned}
J(u) & =\sum_{n \in \mathbb{Z}} a(n) G(u(n)) \\
& \leq \sum_{n \in \mathbb{Z}} a(n)|G(u(n))| \\
& \leq \sum_{n \in \mathbb{Z}} a(n)\left[\frac{\epsilon}{4\left(1+\|a\|_{\infty}\right)}|u(n)|^{2}+T_{\epsilon}|u(n)|^{3}\right] \\
& \leq \frac{\epsilon}{4 \varrho_{2}}\|u\|^{2}+\varrho_{2}^{-1}\left[\left(\varrho_{2}+4 \varrho_{1}\right) \varrho_{2}\right]^{\frac{-1}{4}} T_{\epsilon}\|a\|_{\infty}\|u\|^{3}, \quad \forall u \in X .
\end{aligned}
$$


Thus, for each $u \in X \backslash\{0\}$,

$$
\begin{aligned}
\frac{J(u)}{\Phi(u)} & \leq \frac{\frac{\epsilon}{4 \varrho_{2}}\|u\|^{2}+\varrho_{2}^{-1}\left[\left(\varrho_{2}+4 \varrho_{1}\right) \varrho_{2}\right]^{\frac{-1}{4}} T_{\epsilon}\|a\|_{\infty}\|u\|^{3}}{\frac{1}{2}\|u\|^{2}} \\
& \leq \frac{\epsilon}{2 \varrho_{2}}+2 \varrho_{2}^{-1}\left[\left(\varrho_{2}+4 \varrho_{1}\right) \varrho_{2}\right]^{\frac{-1}{4}} T_{\epsilon}\|a\|_{\infty}\|u\| .
\end{aligned}
$$

Taking the 'limsup' of the above estimation when $u \rightarrow 0$, the arbitrariness of $\epsilon$ gives the required inequality.

Lemma 3.2 $\limsup \sup _{\|u\| \rightarrow \infty} \frac{J(u)}{\Phi(u)} \leq 0$.

Proof By $\left(\mathrm{F}_{5}\right)$ and $\left(\mathrm{F}_{6}\right)$, for every $\epsilon>0$. there exists $\eta_{\epsilon} \in(0,1)$ such that

$$
|\nabla G(u)| \leq \frac{\epsilon}{2\left(1+\|a\|_{\infty}\right)}|u| \quad \text { for all }|u| \geq \eta_{\epsilon}^{-1} \text { or }|u| \leq \eta_{\epsilon}
$$

Since $G \in C^{1}\left(\mathbb{R}^{N}, \mathbb{R}\right)$, there exists a constant $C_{\epsilon}>0$ such that

$$
\frac{|\nabla G(u)|}{|u|^{\gamma}} \leq C_{\epsilon} \quad \text { for all }|u| \in\left[\eta_{\epsilon}, \eta_{\epsilon}^{-1}\right]
$$

where $\gamma$ is given in $\left(\mathrm{F}_{4}\right)$. By virtue of (3.2), (3.3), and $\left(\mathrm{F}_{3}\right)$, we have

$$
|G(u)| \leq \frac{\epsilon}{2\left(1+\|a\|_{\infty}\right)}|u|^{2}+C_{\epsilon}|u|^{\gamma+1}
$$

By $\left(\mathrm{F}_{4}\right),(3.4),(2.3)$, and Hölder's inequality, we obtain

$$
\begin{aligned}
J(u) & =\sum_{n \in \mathbb{Z}} a(n) G(u(n)) \\
& \leq \sum_{n \in \mathbb{Z}} a(n)|G(u(n))| \\
& \leq \sum_{n \in \mathbb{Z}} a(n)\left[\frac{\epsilon}{2\left(1+\|a\|_{\infty}\right)}|u(n)|^{2}+C_{\epsilon}|u(n)|^{\gamma+1}\right] \\
& \leq \frac{\epsilon}{2 \varrho_{2}}\|u\|^{2}+C_{\epsilon}\|a\|_{\frac{2}{1-\gamma}}\|u\|_{2}^{\gamma+1} \\
& \leq \frac{\epsilon}{2 \varrho_{2}}\|u\|^{2}+\varrho_{2}^{-\frac{1+\gamma}{2}} C_{\epsilon}\|a\|_{\frac{2}{1-\gamma}}\|u\|^{\gamma+1}, \quad \forall u \in X .
\end{aligned}
$$

Thus, for each $u \in X \backslash\{0\}$,

$$
\begin{aligned}
\frac{J(u)}{\Phi(u)} & \leq \frac{\frac{\epsilon}{2 \varrho_{2}}\|u\|^{2}+\varrho_{2}^{-\frac{1+\gamma}{2}} C_{\epsilon}\|a\|_{\frac{2}{1-\gamma}}\|u\|^{\gamma+1}}{\frac{1}{2}\|u\|^{2}} \\
& \leq \frac{\epsilon}{\varrho_{2}}+2 \varrho_{2}^{-\frac{1+\gamma}{2}} C_{\epsilon}\|a\|_{\frac{2}{1-\gamma}}\|u\|^{\gamma-1} .
\end{aligned}
$$

Taking the 'lim sup' of the above estimation when $\|u\| \rightarrow \infty$, the arbitrariness of $\epsilon$ gives the required inequality. 
Lemma $3.3 \sup _{\Phi(u)>0} \frac{J(u)}{\Phi(u)}>0$.

Proof Since $a$ is a non-negative, non-zero function, there exists $n_{1} \in \mathbb{Z}$ such that $a\left(n_{1}\right)>0$. Define

$$
v(n)= \begin{cases}\zeta, & n=n_{1} \\ 0, & n \neq n_{1}\end{cases}
$$

Obviously, $\Phi(v)=\frac{1}{2}\|v\|>0$. It follows from (3.5), $\left(\mathrm{F}_{3}\right)$, and $\left(\mathrm{F}_{7}\right)$ that

$$
J(v)=\sum_{n \in \mathbb{Z}} a(n) G(v(n))=a\left(n_{1}\right) G(\zeta)>0 .
$$

Hence we have

$$
\alpha_{2}=\sup _{\Phi(u)>0} \frac{J(u)}{\Phi(u)}>0 .
$$

The proof is complete.

Lemma 3.4 $\lambda_{1}=\frac{1}{\alpha_{2}} \geq \frac{1}{2 D \varrho_{2}^{-1}\|a\|_{\infty}}$, where $\alpha_{2}$ is defined in Theorem 2.1 and $D=\max _{u \neq 0} \frac{G(u)}{|u|^{2}}$.

Proof It follows from $\left(\mathrm{F}_{4}\right)$ and (2.3) that

$$
\begin{aligned}
J(v) & =\sum_{n \in \mathbb{Z}} a(n) G(v(n)) \\
& \leq D\|a\|_{\infty}\|v\|_{2}^{2} \\
& \leq D \varrho_{2}^{-1}\|a\|_{\infty}\|v\|^{2}
\end{aligned}
$$

By (3.7), we have

$$
\alpha_{2}=\sup _{\Phi(u)>0} \frac{J(u)}{\Phi(u)} \leq 2 D \varrho_{2}^{-1}\|a\|_{\infty}
$$

which ends the proof.

Now we give the proof of Theorem 1.1.

Proof Obviously, $X$ is a separable, reflexive and uniformly convex Banach space. It follows from Lemmas 2.3, 2.4, and Lemmas 3.1-3.4 that $\Phi, J$, and $\Psi$ satisfy all conditions of Theorem 2.1. Thus, for each $\lambda>\lambda_{1} \geq \frac{1}{2 D \varrho_{2}^{-1}\|a\|_{\infty}}$, there is $\sigma>0$ with the property that for each $\mu \in[0, \sigma], I$ has at least three solutions in $X$ whose norms are less than $N_{1}$. It is easy to see that 0 is a solution of (1.1). Hence, system (1.1) has at least two nontrivial homoclinic solutions.

Now we give the proof of Theorem 1.2.

Proof Obviously, $X$ be a reflexive real Banach space. With similar arguments to those used in the proofs of Lemmas 2.3 and 2.4, we can see that $\Phi, J: X \rightarrow R$ are two Gâteaux differentiable functionals such that $\Phi$ is sequentially weakly lower semicontinuous, continuous, 
and coercive and $J$ is sequentially weakly continuous. Our aim is to apply part (b) of Theorem 2.2.

Next, we show that $\gamma_{1}<+\infty$. Let $\left\{\eta_{k}\right\}$ be a sequence of positive numbers such that $\lim _{k \rightarrow \infty} \eta_{k}=+\infty$ and

$$
\lim _{k \rightarrow \infty} \frac{\max _{|u| \leq \eta_{k}} G(u)}{\eta_{k}^{2}}=\liminf _{\eta \rightarrow \infty} \frac{\max _{|u| \leq \eta} G(u)}{\eta^{2}} .
$$

Take $r_{k}=\frac{\eta_{k}^{2} \sqrt{\left(\varrho_{2}+4 \varrho_{1}\right) \varrho_{2}}}{2}$ for all $k \in \mathbb{N}$. By $(2.1)$, one has

$$
\|v\|_{\infty} \leq \eta_{k}
$$

for any $v \in X$ with $\|v\|^{2}<2 r_{k}$. In view of (3.8), (3.9), and $\Phi(0)=J(0)=0$, we have

$$
\begin{aligned}
\varphi_{1}\left(r_{k}\right) & =\inf _{u \in \Phi^{-1}\left(\left(-\infty, r_{k}\right)\right)} \frac{\sup _{v \in \Phi^{-1}\left(\left(-\infty, r_{k}\right)\right)} J(v)-J(u)}{r_{k}-\Phi(u)} \\
& \leq \frac{\sup _{v \in \Phi^{-1}\left(\left(-\infty, r_{k}\right)\right)} J(v)}{r_{k}} \\
& \leq \frac{2\|a\|_{1}}{\sqrt{\left(\varrho_{2}+4 \varrho_{1}\right) \varrho_{2}}} \frac{\max _{|u| \leq \eta_{k}} G(u)}{\eta_{k}^{2}} .
\end{aligned}
$$

Thus, by $\left(\mathrm{F}_{10}\right)$, we have

$$
0 \leq \gamma_{1} \leq \frac{2\|a\|_{1}}{\sqrt{\left(\varrho_{2}+4 \varrho_{1}\right) \varrho_{2}}} \liminf _{\eta \rightarrow \infty} \frac{\max _{|u| \leq \eta} G(u)}{\eta^{2}}<+\infty .
$$

In view of $\left(\mathrm{F}_{10}\right)$, we can consider the interval $\Omega$. A simple reasoning related to (3.10) and $\left(\mathrm{F}_{10}\right)$ shows that

$$
\Omega \subseteq\left(0, \frac{1}{\gamma_{1}}\right)
$$

Now, we will verify that $I$ is unbounded from below. It follows from the choice of $\lambda$ that there exists a sequence $\left\{\rho_{k}\right\}$ in $\mathbb{R}^{N}$ with $\left|\rho_{k}\right| \rightarrow \infty$ such that

$$
\frac{G\left(\rho_{k}\right)}{\left|\rho_{k}\right|^{2}}>\frac{2 l_{1}+l_{2}}{2 \lambda a\left(n_{0}\right)}, \quad \forall k \in \mathbb{N} .
$$

We define the function

$$
v_{k}(n)= \begin{cases}\rho_{k}, & n=n_{0}, \\ 0, & n \neq n_{0} .\end{cases}
$$

It is easy to see that $v_{k} \in X$. By $\left(\mathrm{F}_{10}\right),\left(\mathrm{F}_{11}\right),(3.11)$, and (3.12), we have

$$
\begin{aligned}
I\left(v_{k}\right) & =\Phi\left(v_{k}\right)-\lambda J\left(v_{k}\right) \\
& =\frac{1}{2}\left[\left(\left(p\left(n_{0}\right)+p\left(n_{0}+1\right)\right) \rho_{k}, \rho_{k}\right)+\left(L\left(n_{0}\right) \rho_{k}, \rho_{k}\right)\right]-\lambda \sum_{n \in \mathbb{Z}} a(n) G\left(v_{k}(n)\right)
\end{aligned}
$$




$$
\begin{aligned}
& =\frac{1}{2}\left[\left(\left(p\left(n_{0}\right)+p\left(n_{0}+1\right)\right) \rho_{k}, \rho_{k}\right)+\left(L\left(n_{0}\right) \rho_{k}, \rho_{k}\right)\right]-\lambda a\left(n_{0}\right)\left|\rho_{k}\right|^{2} \frac{G\left(\rho_{k}\right)}{\left|\rho_{k}\right|^{2}} \\
& \leq\left|\rho_{k}\right|^{2}\left(\frac{1}{2}\left(2 l_{1}+l_{2}\right)-\lambda a\left(n_{0}\right) \frac{G\left(\rho_{k}\right)}{\left|\rho_{k}\right|^{2}}\right) .
\end{aligned}
$$

Combining (3.11) and (3.13), we find that $I$ is unbounded from below, that is, $I$ has no global minimum.

By part (b) of Theorem 2.2, there is a sequence $\left\{u_{k}\right\}$ of critical points (local minima) of $\Phi-\lambda J$ such that $\lim _{k \rightarrow+\infty} \Phi\left(u_{k}\right)=+\infty$. Hence, system (1.1) possesses an unbounded sequence of homoclinic solutions.

\section{Example}

In order to illustrate Theorem 1.2, we give an example.

Example 4.1 Consider the following problem for the case of $N=1$ :

$$
-\Delta^{2} u(n-1)+u(n)=\lambda a(n) g(u(n)), \quad n \in \mathbb{Z},
$$

where $p=L=1, a(n)=\frac{1}{1+n^{2}}$, and

$$
g(u)= \begin{cases}\pi(k+1) !\left[((k+1) !)^{2}-(k !)^{2}\right] \sin \left(2 \pi(k+1) !\left(u-d_{k}\right)\right), & \text { if } u \in \bigcup_{k \in \mathbb{N}}\left[d_{k}, e_{k}\right], \\ 0, & \text { otherwise, }\end{cases}
$$

with $d_{k}=\frac{k !(k+2) !-1}{4(k+1) !}$ and $e_{k}=\frac{k !(k+2) !+1}{4(k+1) !}$ for every $k \in \mathbb{N}$.

Define

$$
G(u)=\int_{0}^{u} g(s) d s
$$

for all $u \in \mathbb{R}$. It is easy to verify that

$$
g(u) \geq 0, \quad \forall u \in \mathbb{R},
$$

moreover, for any $k \in \mathbb{N}$,

$$
\int_{d_{k}}^{e_{k}} g(s) d s=((k+1) !)^{2}-(k !)^{2} .
$$

By (4.2) and (4.3), we see that $G \in C^{1}(\mathbb{R})$ is nondecreasing. For any $k \in \mathbb{N}$ one has

$$
\begin{aligned}
G\left(d_{k}\right) & =\int_{0}^{d_{k}} g(s) d s \\
& =\int_{0}^{e_{k-1}} g(s) d s \\
& =\sum_{j=0}^{k-1} \int_{d_{j}}^{e_{j}} g(s) d s \\
& =\sum_{j=0}^{k-1}\left[((j+1) !)^{2}-(j !)^{2}\right] \\
& =(k !)^{2}-1
\end{aligned}
$$


and

$$
\begin{aligned}
G\left(e_{k}\right) & =\int_{0}^{e_{k}} g(s) d s \\
& =\sum_{j=0}^{k}\left[((j+1) !)^{2}-(j !)^{2}\right] \\
& =((k+1) !)^{2}-1 .
\end{aligned}
$$

Thus,

$$
\lim _{k \rightarrow \infty} \frac{G\left(d_{k}\right)}{d_{k}^{2}}=0, \quad \lim _{k \rightarrow \infty} \frac{G\left(e_{k}\right)}{e_{k}^{2}}=16,
$$

and in fact, since $\lim _{u \rightarrow-\infty} \frac{G(u)}{u^{2}}=0$, it is a simple computation to verify that

$$
\liminf _{\eta \rightarrow \infty} \frac{\max _{|u| \leq \eta} G(u)}{\eta^{2}}=\liminf _{\eta \rightarrow \infty} \frac{G(\eta)}{\eta^{2}}=0, \quad \limsup _{\eta \rightarrow \infty} \frac{G(\eta)}{\eta^{2}}=16
$$

Applying Theorem 1.2, then, for $\lambda>\frac{3}{32}$, problem (4.1) has an unbounded sequence of homoclinic solutions.

Remark 4.1 In Example 4.1, one cannot obtain the existence of homoclinic solutions in $[20,22,24,25,28,29,31]$. But in this paper, we obtain the existence of infinitely many homoclinic solutions.

\section{Competing interests}

The authors declare that they have no competing interests.

Authors' contributions

All authors contributed equally to the writing of this paper. All authors read and approved the final manuscript.

\section{Author details}

'School of Mathematics and Statistics, Central South University, Changsha, Hunan 410083, People's Republic of China. ${ }^{2}$ School of Mathematics and Physics, University of South China, Hengyang, Hunan 421001, People's Republic of China.

\section{Acknowledgements}

The second author was supported by the Doctor Priming Fund Project of University of South China (2014XQD13). The authors would like to thank the referees for their valuable suggestions and comments, which led to improvement of the manuscript.

Received: 9 November 2014 Accepted: 17 June 2015 Published online: 02 July 2015

\section{References}

1. Chen, HW, He, ZM: Infinitely many homoclinic solutions for a class of second-order Hamiltonian systems. Adv. Differ Equ. 2014, 161 (2014)

2. Caldiroli, P, Montecchiari, P: Homoclinic orbits for second order Hamiltonian systems with potential changing sign. Commun. Appl. Nonlinear Anal. 1(2), 97-129 (1994)

3. Carrião, PC, Miyagaki, OH: Existence of homoclinic solutions for a class of time-dependent Hamiltonian systems J. Math. Anal. Appl. 230, 157-172 (1999)

4. Coti Zelati, V, Ekeland, I, Séré, E: A variational approach to homoclinic orbits in Hamiltonian systems. Math. Ann. 288(1), 133-160 (1990)

5. Coti Zelati, V, Rabinowitz, PH: Homoclinic orbits for second order Hamiltonian systems possessing superquadratic potentials. J. Am. Math. Soc. 4, 693-727 (1991)

6. Ding, $\mathrm{YH}$ : Existence and multiplicity results for homoclinic solutions to a class of Hamiltonian systems. Nonlinear Anal. 25, 1095-1113 (1995)

7. Izydorek, M, Janczewska, J: Homoclinic solutions for a class of the second order Hamiltonian systems. J. Differ. Equ. 219, 375-389 (2005) 
8. Omana, W, Willem, M: Homoclinic orbits for a class of Hamiltonian systems. Differ. Integral Equ. 5, 1115-1120 (1992)

9. Paturel, E: Multiple homoclinic orbits for a class of Hamiltonian systems. Calc. Var. Partial Differ. Equ. 12, 117-143 (2001)

10. Rabinowitz, PH: Homoclinic orbits for a class of Hamiltonian systems. Proc. R. Soc. Edinb. Sect. A 114, 33-38 (1990)

11. Rabinowitz, PH, Tanaka, K: Some results on connecting orbits for a class of Hamiltonian systems. Math. Z. 206, 473-499 (1991)

12. Salvatore, A: Homoclinic orbits for a special class of nonautonomous Hamiltonian systems. Nonlinear Anal. 30 4849-4857 (1997)

13. Sun, J, Chen, H, Nieto, JJ: Homoclinic solutions for a class of subquadratic second-order Hamiltonian systems. J. Math. Anal. Appl. 373, 20-29 (2011)

14. Zhang, Q, Liu, C: Infinitely many homoclinic solutions for second order Hamiltonian systems. Nonlinear Anal. 72 894-903 (2010)

15. Zhang, ZH, Yuan, R: Homoclinic solutions for a class of non-autonomous subquadratic second-order Hamiltonian systems. Nonlinear Anal. 71, 4125-4130 (2009)

16. Zou, WM, Li, SJ: Infinitely many homoclinic orbits for the second-order Hamiltonian systems. Appl. Math. Lett. 16, 1283-1287 (2003)

17. Agarwal, RP: Difference Equations and Inequalities: Theory, Methods, and Applications, 2nd edn. Dekker, New York (2000)

18. Ahlbrandt, CD, Peterson, AC: Discrete Hamiltonian Systems: Difference Equations, Continued Fraction and Riccati Equations. Kluwer Academic, Dordrecht (1996)

19. Chen, HW, He, ZM: Homoclinic solutions for second order discrete Hamiltonian systems with superquadratic potentials. J. Differ. Equ. Appl. 19, 1147-1160 (2013)

20. Chen, HW, He, ZM: Infinitely many homoclinic solutions for second-order discrete Hamiltonian systems. J. Differ. Equ. Appl. 19, 1940-1951 (2013)

21. Chen, HW, He, ZM: Homoclinic solutions for second-order discrete Hamiltonian systems with asymptotically quadratic potentials. Math. Methods Appl. Sci. 37, 2451-2462 (2014)

22. Chen, P: Existence of homoclinic orbits in discrete Hamiltonian systems without Palais-Smale condition. J. Differ. Equ. Appl. 19, 1781-1794 (2013)

23. Deng, XQ, Chen, G: Homoclinic orbits for second order discrete Hamiltonian systems with potentials changing sign. Acta Appl. Math. 103, 301-314 (2008)

24. Lin, XY, Tang, XH: Existence of infinitely homoclinic orbits in discrete Hamiltonian systems. J. Math. Anal. Appl. 373 59-72 (2011)

25. Ma, MJ, Guo, ZM: Homoclinic orbits for second order self-adjoint difference equations. J. Math. Anal. Appl. 323(1), 513-521 (2006)

26. Ma, MJ, Guo, ZM: Homoclinic orbits and subharmonics for nonlinear second order difference equations. Nonlinear Anal. 67, 1737-1745 (2007)

27. Tang, $\mathrm{XH}$, Chen, J: Infinitely many homoclinic orbits for a class of discrete Hamiltonian systems. Adv. Differ. Equ. 2013, $242(2013)$

28. Tang, XH, Lin, XY: Existence and multiplicity of homoclinic solutions for second-order discrete Hamiltonian systems with subquadratic potential. J. Differ. Equ. Appl. 17, 1617-1634 (2011)

29. Tang, XH, Lin, XY: Infinitely many homoclinic orbits for discrete Hamiltonian systems with subquadratic potential. J. Differ. Equ. Appl. 19, 796-813 (2013)

30. Tang, XH, Lin, XY, Xiao, L: Homoclinic solutions for a class of second order discrete Hamiltonian systems. J. Differ. Equ. Appl. 16, 1257-1273 (2010)

31. Zhang, X, Shi, YM: Homoclinic orbits of a class of second-order difference equations. J. Math. Anal. Appl. 396, 810-828 (2012)

32. Ricceri, B: A further three critical points theorem. Nonlinear Anal. 71, 4151-4157 (2009)

33. Ricceri, B: A general variational principle and some of its applications. J. Comput. Appl. Math. 113, 401-410 (2000)

34. Zeidler, E: Nonlinear Functional Analysis and Its Applications, vol. 2. Springer, Berlin (1990)

\section{Submit your manuscript to a SpringerOpen ${ }^{\ominus}$ journal and benefit from:}

- Convenient online submission

Rigorous peer review

Immediate publication on acceptance

- Open access: articles freely available online

- High visibility within the field

- Retaining the copyright to your article 